unter denen der Bogen brennt. Die Kommission hat daher beschlossen, den für die internationalen Standards zu benutzenden Bogen, wie folgt, zu normieren: Der Bogen ist mit Gleichstrom von 220 Volt und 6 Ampère für $\lambda>400 \mu \mu$, 4 Ampère für $\lambda<400 \mu \mu \mathrm{zu}$ betreiben. Es ist das $2 \mathrm{~mm}$ lange Stuick aus der Mitte eines Bogens von $6 \mathrm{~mm}$ Länge zwischen Stäben von $7 \mathrm{~mm}$ Stärke, positiver Pol oben, zu verwenden.

Bei der Bestimmung der Sonnenrotation aus den Radialgeschwindigkeiten des Sonnenrandes haben sich eigentümliche systematische Unterschiede zwischen verschiedenen Linien und Beobachtern gezeigt, welche die Kommission zu dem Beschluß geführt haben, zunächst einmal eine allseitige Beobachtung von Io ausgewählten Linien zwischen 4220 und $4280 \AA$. E. am Sonnenäquator zu empfehlen.

Zur nächstjährigen Sonnenfinsternis sind bereits 19 verschiedene Expeditionen angemeldet, die sich fast über die ganze Strecke vom nördlichen Schweden bis zur Krim verteilen werden. Herr Donitch hat die Organisation und die Platzverteilung in Rußland übernommen.

Potsdam, I9I3 August I 4.
Herr Riccó berichtete über seine dreißigjährigen Protuberanzenbeobachtungen, aus denen sich eine ständige Abnahme der Eruptionstätigkeit - natürlich von der I I-jährigen Periode überlagert - ergibt.

Eine neubegründete Subkommission für visuelle Beobachtung der Sonnenatmosphäre traf Festsetzungen üher die Zählweise der Positionswinkel der Protuberanzen und über die Bezeichnung der Stärke der Protuberanzen.

Herr St.Fohn berichtete über seine eingehenden Beobachtungen der zuerst von Evershed bemerkten radialen Bewegungen in und um Sonnenflecken.

Die Kommission für Klassifikation der Sternspektren empfahl bis auf weiteres den Gebrauch des Harvardsystems. Sterne, die genau vom Typus A, B usw. sind, sollen dabei mit $\mathrm{A}_{0}, \mathrm{~B}_{0}$ usw. bezeichnet werden, während $z$. $\mathrm{B}$. die Bezeichnung $A$ ohne Index eine rohere Klassifikation bedeuten soll.

Die Berliner Akademie der Wissenschaften wurde in die Union aufgenommen. Die nächste Tagung ( 1916$)$ soll in Rom stattfinden.

\title{
Bemerkungen zu den Eigenbewegungen auf Jupiter 1913.
}

Ich werde auf Latimer $\mathcal{F}$. Wilsons Mitteilungen hierüber (in Engl. Mech. and W. of Sc. vom 8. August) aufmerksam gemacht und bemerke dazu, daß in der Tat zwei ganz auffallend isolierte und dunkle Flecken am S-Rande des SEB ebenso auffallende retrograde Bewegungen ausführen. Die Flecken sind sich fast gleich an Gestalt und Größe und ahmen fast genau die Form der "Violin *- und "Granat " Flecken im NEB von $1896-98$ nach. Zunächst sah man, wie das vorangehende Ende des "Schleiers", der die STrZ zu einem großen Teile ausfüllt, den zweiten (folgenden) Fleck überholte: Mitte Mai I9I3 lag er um eine ganze Eigenlänge vor dem Schleier, am r. Juli stand nur noch die Hälfte des Flecks vor der scharfen Begrenzung des Schleiers und am I I. August war der Fleck schon der Bucht näher gelegen als dem Schleieranfang: Dieser letztere wurde bisher häufig beobachtet und es konnten 20 Mittelwerte gebildet werden. Sie zeigen, daß der Schleier zwischen April zo und Aug. I I eine ziemlich gleichmäßige tägliche Beschleunigung von 0.327 besaß. Die Bucht $*$, deren Beobachtung 20 Mittelwerte des vorangehenden Randes und 16 des folgenden ergab, hat eine gleichfalls recht stetige Beschleunigung ge-

Landstuhl, I 9 I 3 Aug. 15. habt, aber nur von täglich 0:ro5. Das nachfolgende Schleierende ergab 9 Mittelwerte von bemerkenswerter Unregelmäßigkeit, was an sich nicht ungewöhnlich ist, weil naturgemäß der Schub nach vorwärts (im Westen) Stauung und scharfe Begrenzung erzeugt, das nachfolgende Ende vielfach verzettelt erscheinen kann.

Die beiden oben genannten Einzelflecken haben nun die Sonderbarkeit aufgewiesen, daß sie beide, und zwar der vorangehende bis zum 11 . Mai etwa, der folgende noch vier Wochen länger, abnehmende Beschleunigung, von diesen Zeiten an aber gleichbleibende Verzögerung aufwiesen. Der erste Fleck ist in 92 Tagen 13.2 und der zweite in 64 Tagen $2 I^{\circ} 5$ zurückgeblieben, was für I täglich $0^{\circ}$. 43 und für II den enormen Betrag von 0.336 retrograder Bewegung bedeutet. - Es wird interessant und wichtig sein, festzustellen, wie die Begegnung des Flecks II mit der Bucht selber vor sich geht, die Mitte September zu erwarten wäre. Wenn nicht verschiedene Kräfte zusammenwirken, geht auch dieses zenologisch hochwichtige Ereignis unbeobachtet vorüber, denn wir verfügen leider nur über wenige Observatorien für Planetographie in klimatisch ginstiger Lage.

Ph. Fauth.

\section{Sur l'accélération de la Tache Rouge de Jupiter pendant les conjonctions avec la tache grise tempérée australe.}

Dans le No. 4668 des A. N., M. Antoniadi dit que le I octobre 1902, dans une carte postale adressée au Dr. Crommelin, il avait attiré son attention sur la probabilité que l'accélération de la Tache Rouge, lors de sa conjonction avec la tache grise tempérée australe, pourrait être due à une poussée de cette dernière, et que Mr. Denning, dans "Nature* ( 8 décembre I902), énonça la même hypothèse en toute indépendance, hypothèse qui a été confirmée par les faits.
Je dois ajouter que j'avais déjà publié, dans le No. de septembre r 902 du Bulletin de la Soc. Astr. de France l'hypothèse de la "poussée". Voyez ce No., page 389 , où je dise, en comparant les rotations de la pointe suivante de la Tache Rouge en I90I et I902: "La poussée du courant de protubérances (actuellement, la zone grise tempérée australe) sur la Tache Rouge est donc bien sensible.*

Barcelone, I 9 I 3 Juill. 27.

Fosé Comas Sola. 